\title{
MECHANICALLY DYNAMIC SCAFFOLDS FOR ENHANCED ANTERIOR CRUCIATE LIGAMENT RECONSTRUCTION
}

\author{
J. G Lacombe ${ }^{1}$, M. Cooke², H. Park ${ }^{3}$, J. Ramirez-GarciaLuna², D. Slawaska-Eng' ${ }^{1}$, F. Almarek ${ }^{2}$, DH Rosenzweig², \\ Paul A Martineau ${ }^{2}$ \\ ${ }^{1}$ Experimental Surgery, Faculty of Medicine, McGill University, Montreal, Canada \\ ${ }^{2}$ Division of Orthopaedic Surgery, McGill University Health Centre, Montreal, Canada \\ Mining and Materials Engineering, McGill University, Montreal, Canada
}

\begin{abstract}
Close to a quarter of a million ACL injuries occur each year in Canada and the United States. These injuries can result in reduced functional performance and are associated with long-term clinical conditions that include meniscal tears, chondral lesions and an increased risk of early onset osteoarthritis (OA). Surgical reconstruction is the current gold standard of care for ACL injuries. However, surgical treatment is costly and associated with high risk of post-traumatic OA within two decades of injury. Researchers have explored tissue engineering (TE) to address these shortcomings and have developed degradable graft materials that can guide functional connective tissue formation. However, scaffolds fabricated using TE methods still do not accurately mimic the anisotropic architecture and biomechanical properties of the ACL. Furthermore, the biological graft remodeling process causes significant reductions in tensile properties of the tissue-engineered scaffolds, which adds complexity to the design of such structures. Our hypothesis is that reductions in mechanical properties can be minimized by enclosing the scaffold in an artificial semi-permeable membrane and incubating it in vivo prior to ACL surgical reconstruction. A low-cost ACL scaffold was additively manufactured via fused deposition modeling using LAY-FOMM material (thermoplastic polyurethanes co-polymer with polyvinyl alcohol). Scaffolds replicating the anatomical dimensions of the ACL were printed on a Flashforge Creator Pro 3D Printer and evaluated for mechanical properties such as tensile strength, strain at failure, compression strength and Young's modulus. Second, the scaffolds will be soaked in a solution containing bFGF and TGF- $\beta$ growth factors and seeded with human adipose derived mesenchymal stem cells (ADSCs). The scaffolds will then be placed in a semi-permeable membrane and cells will be investigated for viability via a live/dead analysis and metabolic activity. Cell phenotype will be characterized using western blot and PCR to assess collagen types I and III, fibronectin and tenascin. Lastly, the impact of mechanical stimuli on tissue formation will be investigated using the Mechanoculture T6 bioreactor. There is currently a need for tissue-engineered ACL scaffolds that can appropriately mimic the biomechanical properties of the ACL and guide ligament formation. Due to the nature of the biological processes involved in graft reconstruction, most grafts undergo a significant reduction in tensile properties. The incubation of graft material prior to surgical intervention could maximize mechanical stability and allow patients to go back to regular activities faster with lower risk of recurring injuries.
\end{abstract}

